\title{
Evento de depredación de Caiman crocodilus fuscus por Lontra longicaudis (Carnivora: Mustelidae) en el río Palomino, departamento de La Guajira, Colombia
}

\author{
Oscar D. Medina-Barrios \\ Diana Morales-Betancourt \\ Fundación Omacha, Bogotá, Colombia
}

Fundación Omacha, Bogotá, Colombia. oscardanielmb@hotmail.com

La Nutria Neotropical de río Lontra Longicaudis (Olfers, 1818), es un mamífero semiacuático que ocupa una amplia distribución geográfica, desde México hasta el norte de Argentina (Waldemarin \& Colares 2000, Arellano et al. 2012). Es una especie bastante versátil, que tolera ciertas modificaciones ambientales y puede ocupar áreas cercanas a zonas de actividad antrópica. Sin embargo, las poblacionales de mayor densidad se encuentran en áreas con extensas redes acuáticas, baja contaminación química y carga orgánica, y baja densidad humana (Larivière 1999, Charre-Medellín et al. 2011, Botello et al. 2006). Se alimentan básicamente de peces, crustáceos, moluscos y son consumidores de pequeños mamíferos, aves, reptiles, insectos e inclusive frutos (Larivière 1999, Quadros \& Monteiro-Filho 2000, Gallo-Reynoso et al. 2008, Botello et al. 2006, Charre-Medellín et al. 2011). En esta nota se registra la depredación de una babilla juvenil (Caiman crocodilus fuscus) por parte de la Nutria Neotropical, comportamiento poco común en la especie, además de la modificación de su horario de actividad debido a la creciente demanda turística en el río monitoreado.

Durante el mes de febrero de 2015 se hizo una observación directa in situ de L. longicaudis en el río Palomino (municipio de Dibulla, La Guajira, Colombia) entre las $06 \mathrm{~h} 00$ y las $17 \mathrm{~h} 00$, para documentar varios aspectos de la ecología del comportamiento de la especie. En este periodo se avistaron dos nutrias alimentándose de una babilla juvenil (C. c. fuscus) en un área de vegetación asociada a la ribera y cerca de la desembocadura al mar $\left(11^{\circ} 14^{\prime} 54,7^{\prime \prime} \mathrm{N}, 073^{\circ} 34^{\prime} 05,4^{\prime \prime} \mathrm{W}\right)$ a una altura de $16 \mathrm{msnm}$. Tal comportamiento es poco frecuente en la especie (Figura 1). Este tipo de alimentación observado puede sugerir que la nutria se ve favorecida por la captura de comida en forma oportunista (Kasper et al. 2004, Quadros \& Monteiro Filho 2002) en ambientes intervenidos (como el lugar de la observación; Santos \& Reis 2012), mostrando así su característica generalista (Gallo-Reynoso 1989, Spínola \& Vaughan 1995, Pardini 1998, Macías-Sánchez \& Aranda 1999, Quadros \& Monteiro-Filho 2000).

Se podría inferir que la ingesta de C. c. fuscus se dio de forma casual. Sin embargo, dicho comportamiento puede relacionarse directamente a que la especie eventualmente incluye este reptil en su dieta, ya sea por la facilidad de depredar los individuos juveniles, la importante biomasa que aporta y/o su disponibilidad y abundancia en la zona (Lanski \& Molnar 2003, Morales et al. 2004, Guerrero-Flores et al. 2013).

Es importante notar que estos registros fueron documentados entre las $06 \mathrm{~h} 00$ y las $09 \mathrm{~h} 00$, contrario a lo reportado por otros autores quienes sugieren que la especie muestra mayores picos de actividad al medio día y en las horas crepusculares (Parera 1993, Lariviére 1999). El hecho que desarrollen sus actividades a tempranas horas de la mañana, puede indicar que $L$. longicaudis está modificando sus jornadas de forrajeo debido a la ocurrencia de bañistas en la zona, a partir de las 10h00, los cuales se incrementan hacia las horas de la tarde, según lo apreciado al momento de hacer las estaciones de observación. Este comportamiento puede indicar que la especie presenta cierta tolerancia y adaptabilidad a la presencia humana. Sin embargo, se hace necesario el seguimiento de la especie en este río durante otros períodos a largo plazo, con el fin de documentar los efectos del turismo sobre la especie y así generar los insumos suficientes para realizar los planes de manejo y conservación necesarios y más adecuados para la zona.

\author{
Mammalogy Notes | Notas Mastozoológicas \\ Sociedad Colombiana de Mastozoología \\ Vol. 2 Num. 1 | 2015
}


QUADROS, J. \& L.A. MONTEIRO-FILHO. 2002. Sprainting sites of the Neotropical otter, Lontra longicaudis, in an Atlantic Forest area of Southern Brazil. Mastozoologia Neotropical 9: 39-46.

SANTOS, L.B. \& N.R. REIS. 2012. Use of shelters and marking sites by Lontra longicaudis (Olfers, 1818) in lotic and semilotic environments. Biota Neotrop. 12(1): 199-205. SPÍNOLA, R.M. \& C. VAUGHAN. 1995. Dieta de la nutria neotropical (Lutra longicaudis) en la estación biológica La Selva, Costa Rica. Vida Silvestre Neotropical 4: 125-132. WALDEMARIN, H.F. \& E.P. COLARES. 2000. Utilisation of Resting Sites and Dens by the Neotropical river otter (Lutra longicaudis) in the South of Rio Grande do Sul State, Southern Brazil. IUCN Otter Spec. Group Bull. 17:14-19. 